\title{
Possibilities of Using Graphical and Numerical Tools in the Exposition of Process Capability Assessment Techniques
}

DOI: 10.12776/QIP.V23I2.1219

Josef Tošenovský, Filip Tošenovský

Received: 18 January 2019 Accepted: 17 June 2019 Published: 31 July 2019

\begin{abstract}
Purpose: The paper focuses on how the problem of process capability assessment can be handled when taught, using convenient numerical and graphical means. The contents of the paper results from the authors' own academic and practical experience, which suggested that many important steps are overlooked in the process of selecting and using capability indices.
\end{abstract}

Methodology/Approach: Selected problems in capability assessment are illustrated with suitable examples and graphs.

Findings: The authors' experience is reflected in the paper, aiming to emphasize what matters and how, and what does not. Also, a new capability index is introduced.

Research Limitation/implication: The style in which the problems are analysed may serve as a guide for further studies in the field and capability index applications.

Originality/Value of paper: The paper also contains, aside from specific examples, some more advanced techniques, and is therefore accompanied by software readouts, since computer support is required in such cases.

Category: Conceptual paper

Keywords: process capability; capability index selection; process robustness 


\section{INTRODUCTION}

This paper presents our experience with education in the field of process capability assessment. It is intended for everyone who plans to dedicate their career to process evaluation and is willing to practise it credibly in the future. During courses run in a selected corporate sector, we encountered many imperfections, ambiguities and problems that we tried to clarify in the paper in an illustrative way, using computational examples and graphical tools. We would like to pass on some of what we have learnt in this process to the interested reader. The paper consists of several sections that cover both univariate and multivariate capability indices. Since univariate indices are more popular, the emphasis is placed on them, and diverse practical situations are dealt with based on how the problem at hand is defined. The text begins with conditions that should be met in order for a capability index to work properly, and then moves on to the problem of selection of an appropriate index. The latter, in particular, has to do with the specific situation the index user happens to find himself in. Further, the paper also pays attention to the evaluation of capability indices and the term "robustness" that has its place in the theory of process capability assessment for all those who seek excellence (Zgodavova and Slimak, 2008).

\section{PROCESS CAPABILITY ASSESSMENT AND CONDITIONS}

capability index formula being applied without seeing the broader picture of doing so. We shall therefore start our pedagogical journey with the interpretation of formulas. Each capability index is given by a formula that can only be used under certain conditions. If $B$ denotes such a formula, we can view its use as a result of the implication $A \Rightarrow B$, where $A$ stands for the conditions. If the conditions $A$ hold, the formula $B$ is true. The formula will certainly work when the conditions are met. It may also happen to work when the conditions fail to hold, but it may not work, so it is advisable to avoid using it when the conditions are not met because we are not certain what will happen. Thus, knowing the conditions is fundamental to putting the indices to use.

The practice, it appears, is such that only one specific capability index is applied, whatever the process, or such usage is even required in this context. These situations usually concern the $C_{p k}$ capability index. But an application of $C_{p k}$, or any other index, is linked to the conditions for its use, as we already know. Let us start with the conditions. To make them systematic and facilitate orientation within their framework, we now divide them into two categories. We work with:

- general conditions,

- specific conditions.

The general conditions are those that must always be met, regardless of the capability index used. If they do not hold, one cannot continue to assess process 
capability with an index. The problem must be removed before any capability assessment takes place.

The specific conditions are index-specific extra conditions which must hold in addition to the general conditions. These conditions usually accompany the definition of a capability index. Both the general and specific conditions should be verified with statistical tests, or also in combination with suitable graphical methods. Nowadays there are capability indices suitable basically for any situation, so there is no reason to improvise and use a specific index outside the conditions that define its application. In this context, it is perhaps necessary to say that when an organization strictly requires that its suppliers calculate a specific index, such as $C_{p k}$, regardless of whether the index matches the suppliers' production environment, it does not boost its credibility. In these cases, one may ask what good such an index is and how serious the customer is about it. Since, as is known, the customer must be complied with, one way of proceeding is to present the required index with a supplementary explanation what the specific environment of the organization is, and what adequate index should be applied. Such an index should be announced, and the attention should be drawn to the potential discrepancy between the two indices and the unreliability of the required index. Companies often do not comply, if assessed by the required index, but do comply with the standards set by the proper index!

The general conditions are related to the evaluated process, data and tolerance. They are:

- The process is stable.

- The data on the process are independent, without outliers, sufficient in size.

- The tolerance is specified correctly.

If any of the conditions fails, it is advisable not to calculate the capability index. Otherwise, the resulting value is unreliable. It is overestimated or underestimated, depending on which condition failed to hold. The value of the index can also be meaningless. Note that normality of the data is not among the general conditions. Process capability can be evaluated without normality. In relation to these conditions, many questions arise that the user of an index should ask, such as how to verify the validity of the conditions, what exactly happens when they do not hold, or how to proceed in the less favourable situation when they fail. Comparing various scholarly publications on capability assessment, we find out that the set of conditions differs slightly among the authors, however there is definitely a consensus regarding the condition of process stability. This condition is crucial.

A process is stable in the statistical sense of the word when all its monitored quality characteristics lie within the control limits of the corresponding control charts. Of course, this means that control charts must be an established tool in 
organizations. This, however, can be a problem. It seems that many organizations do not know that when control charts are not available, it is possible to verify the process stability easily, fast and reliably with a statistical test. This is true even when more than one quality characteristic is observed for a process. The truth is that such a test is not commonly implemented in statistical software packages, but the situation is not hopeless. The interested reader may find a theoretical exposition of the tests in Holmes and Mergen (1995), its practical use is implemented, for instance, in the computer program Capa (Tošenovský, 2006).

When analysing a process, defining the tolerance for its quality characteristic(s) and gathering data on the process is how the capability assessment begins. What then follows is the selection of an appropriate capability index.

\section{SELECTION OF A CAPABILITY INDEX}

When selecting a univariate index (one quality characteristic observed), verifying normality of the data should be the first step. If the data come from a normal distribution, the next step is to further narrow down the selection so that it is in line with the type of tolerance worked with. Tab. 1 shows the situation, including the selection procedure in the case of non-normal data.

Table 1 - Capability Index Selection Scenarios

\begin{tabular}{|c|c|c|c|}
\hline \multicolumn{4}{|c|}{ One quality characteristic observed } \\
\hline \multicolumn{2}{|c|}{ Normal distribution - tolerance: } & \multicolumn{2}{|r|}{ Non - normal distribution: } \\
\hline Symmetric & $\begin{array}{l}C_{p}, C_{p k}, C_{p m}, C_{p m k} \\
\text { (Kotz and Johnson, 1993) }\end{array}$ & $\begin{array}{l}\text { Mass } \\
\text { production }\end{array}$ & $\begin{array}{l}\text { Special indices } \\
\text { (Pearn and Kotz, 1995; Clements, } \\
\text { 1989) }\end{array}$ \\
\hline Asymmetric & $\begin{array}{l}C_{p m}^{*} \\
\text { (Chan, Cheng and Spiring, 1988) }\end{array}$ & $\begin{array}{l}\text { Unit } \\
\text { production }\end{array}$ & $\begin{array}{l}Q, C_{p T} \\
\text { (Schneider, Pruett and Lagrange, } \\
1996)\end{array}$ \\
\hline One-sided & $\begin{array}{l}C_{p p}, C_{p T} \\
\text { (Phillips, 1995; Schneider, Pruett } \\
\text { and } \quad \text { Lagrange, } \\
\text { Krishnamoorthi, 1990) }\end{array}$ & Attributes & (Bothe, 2000) \\
\hline Unbounded & Modified $C_{p m}, C_{p k}$ & & \\
\hline
\end{tabular}

\subsection{Symmetric Tolerance}

The procedure of assessing capability for the case of symmetric (and asymmetric) tolerances is well-known. Let us note that all the frequently applied indices $C_{p}, C_{p k}, C_{p m}, C_{p m}^{*}$ and $C_{p m k}$ have data normality as their specific condition of use, and for $C_{p}$, there is an additional condition $\mu=T$, i.e. the 
process must be centred. Unlike other indices, $C_{p}$ does not reflect the extent to which the expected value $\mu$ of the quality characteristic complies with the defined target $T$. Thus, it can happen that an uncentered process with small $\sigma$ can have a better $C_{p}$ than a centered process with far higher variability $\sigma$ : for the specifications $\mathrm{LSL}=10, \mathrm{USL}=16, \mathrm{~T}=13$, for instance, the process for which:

a) $\mu=\mathrm{T}=13$ (a perfectly centered process), $\sigma=1$, the index equals 1 ;

b) $\mu=19$ (a process off the target), $\sigma=0.5$, the index equals 2 .

Regarding $C_{p k}$, where data normality is the only specific condition, a lack of centralization can be offset by a reduction in the variability of the quality characteristic, if this is possible (see the example below). It can be used for technologies where there is no problem to adjust both the expected value $\mu$ of the quality characteristic and its variance $\sigma^{2}$. If it is convenient for the producer to keep $\mu$ near one of the tolerance limits, it will follow this strategy. To give an example, for a supplier of sand, for the humidity of which the target value is $T$ and the lower and upper tolerance limits are $L S L$ and $U S L$, respectively, it is convenient to keep the average humidity $\mu$ close to USL (the weight of sand pays) and keep $\sigma^{2}$ at the same time at such a level that the required value of $C_{p k}$ will be fulfilled. In the documentation provided by the customer, it is therefore not enough to state the intended value of $C_{p k}$. The requirement $\mu=T$ should be mentioned, as well. To use specific values, let us assume $U S L=65, L S L=35$ and $T=50$. The expected value is pushed away from the target value, but $\sigma$ is being reduced at the same time (see the values below for the two characteristics). In all the cases, $C_{p k}=1$.

a) $\mu=50, \sigma=5$,

b) $\mu=53, \sigma=4$,

c) $\mu=56, \sigma=3$,

d) $\mu=59, \sigma=2$.

We shall now comment on other situations listed in Tab. 1.

\subsection{Unbounded Tolerance}

This is the case when one of the tolerance limits is unbounded. In tis case, we arrive at the modified versions of $C_{p m}$ and $C_{p k}$. The modified $C_{p m}$ is:

for the case $L S L=-\infty$

$$
C_{p m}^{*}=\frac{\min \{U S L-T, T-(-\infty)\}}{3 \tau}=\frac{U S L-T}{3 \tau}
$$


and for the case $U S L=+\infty$

$$
C_{p m}^{*}=\frac{\min \{+\infty-T, T-L S L\}}{3 \tau}=\frac{T-L S L}{3 \tau}
$$

where

$$
\tau^{2}=n^{-1} \sum_{i}\left(x_{i}-T\right)^{2}
$$

If no $T$ is defined, one may modify the $C_{p k}$ index. The modified index is calculated as follows:

for the case $L S L=-\infty$

$$
C_{p k}=\frac{U S L-\mu}{3 \sigma}
$$

and for the case $U S L=+\infty$

$$
C_{p k}=\frac{\mu-L S L}{3 \sigma}
$$

\subsection{One-sided Tolerance}

There are situations when the target value $T$ equals one of the tolerance limits $T=U S L$ or $T=L S L$. In technical documentation, the situation is denoted as $T_{+d}^{-0}$ or $T_{0}^{-d}$, when $\mathrm{T}=\mathrm{LSL}$ or T $=\mathrm{USL}, d$ being the tolerance (Schneider, Pruett and Lagrange, 1996). Strictly speaking, this is not an asymmetric tolerance. It is not reasonable to use $C_{p k}$ for this type of tolerance, as we shall see. The situation is illustrated in Fig. 1 and Fig. 2. Let us recall that the objective of capability assessment is to make a judgement on process centralization and variability. Fig. 1 shows the case $T=L S L$.

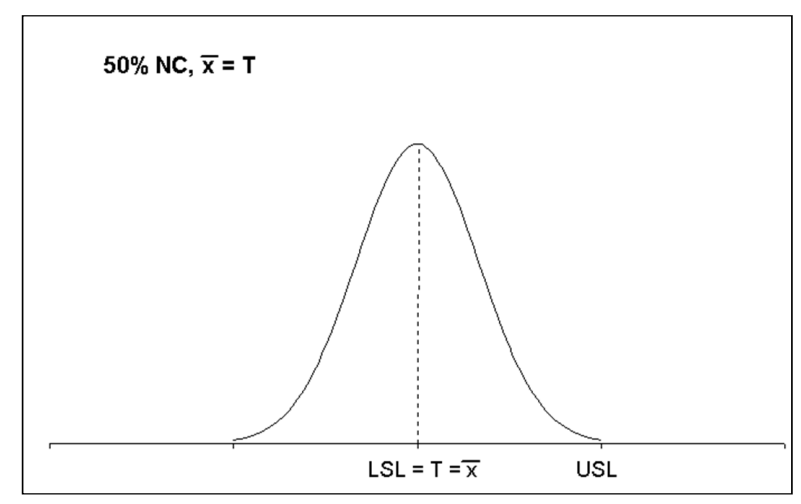

Figure $1-\bar{x}=$ T but $N C=50 \%$ 
Assuming that the process is centred, or the sample average of the observed quality characteristic is equal to the target value, then fifty per cent of the process output will be flawed, in other words, it will represent nonconforming products (NC). If the process is not centred, but its output is within the tolerance limits (no flawed products), the target value will never be achieved, as shown in Fig. 2.

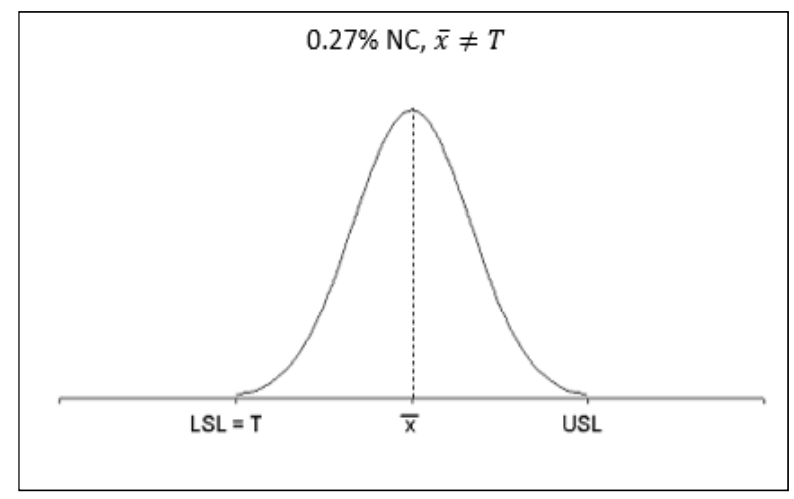

Figure $2-N C=0.27 \%$, but $\bar{x} \neq T$

It is not possible to comply with both requirements - being centred and minimize NC. Producers proceed in such cases logically - their process follows the center of the tolerance interval. This explains why there is no point in using $C_{p k}$, which evaluates the amount of centralization, purposefully violated in this case. The $C_{p p}$ and $C_{p T}$ indices, on the contrary, seem very suitable here (Schneider, Pruett and Lagrange, 1996). They can be applied to both the two-sided and one-sided tolerances, regardless of whether the data the indices are calculated from come from a normal distribution. For non-normal data, one can proceed in more than one way. Special indices can be used, or the data may be transformed so that their true distribution is brought closer to normality. A separate category is represented by non-measurable quality characteristics (attributes) and the socalled unit production.

\section{ROBUSTNESS AND ITS SIGNIFICANCE}

After an index is calculated, it should be evaluated (Tab. 2), i.e. a judgement should be made as to whether its value is high enough for the given amount of data, from which it was calculated, and whether there is enough room for a potential process deterioration, i.e. how robust the process is. 
The property that when the average of a process quality characteristic deviates from the target value, yet it doesn't lead to a higher number of $N C$ products, is called process robustness. It can be quantified by the equation:

$$
R=3 \sigma\left(C_{p}-1\right)
$$

$R$ describes the distance by which the average $\mu$ can move away from the target value $T$ without the process losing its capability. The distance is expressed as a multiple of $\sigma$. To give an example of this interpretation, let $C_{p}=1.33$. Then $(U S L-L C L) / 6 \sigma=1.33 \Rightarrow U S L-L S L=7.98 \sigma \cong 8 \sigma$. The length of the tolerance interval, a multiple of $\sigma$, is roughly $8 \sigma$. The length containing for a normally distributed quality characteristic $99.73 \%$ of its values is $6 \sigma$. Calculating the robustness, we have $R=3 \sigma(1.33-1)=\sigma$. Looking at Fig. 3, we see the average can shift from $T$ by $\sigma$ without increasing palpably $N C$.

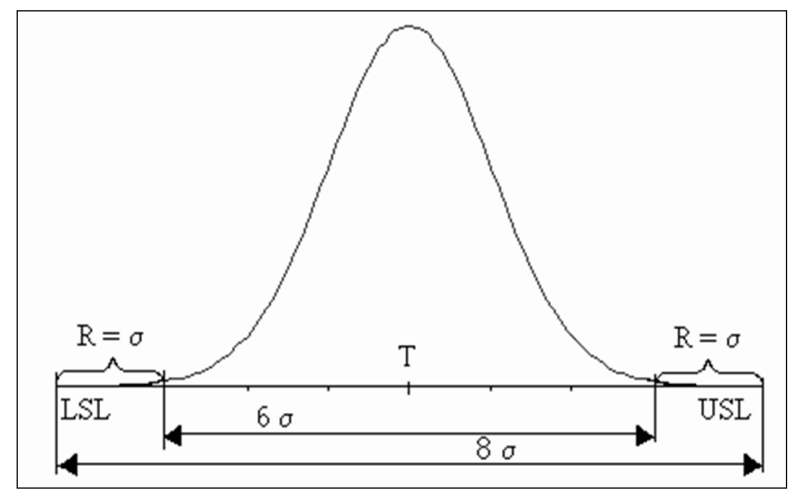

Figure $3-$ Robustness $R=\sigma$

To give another example, if $C_{p}=1.67$, then $(U S L-L C L) / 6 \sigma=1.67 \Rightarrow U S L-L S L=9.96 \sigma \cong 10 \sigma$ and $R=3 \sigma(1.67-1) \approx 2 \sigma$.

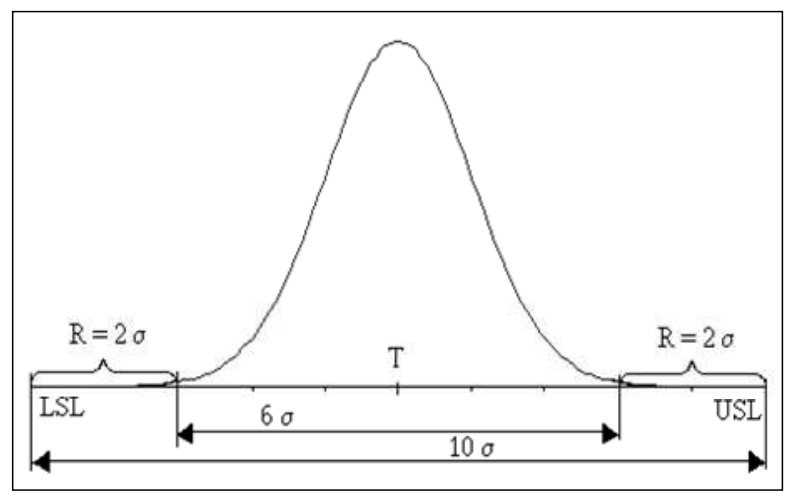

Figure 4 - Robustness $R=2 \sigma$ 
For $C_{p}=1, R=3 \sigma(1-1)=0$ (Fig. 5). $R=0$ means that even a very small deterioration leads to process incapability (to exceeding the tolerance limits).

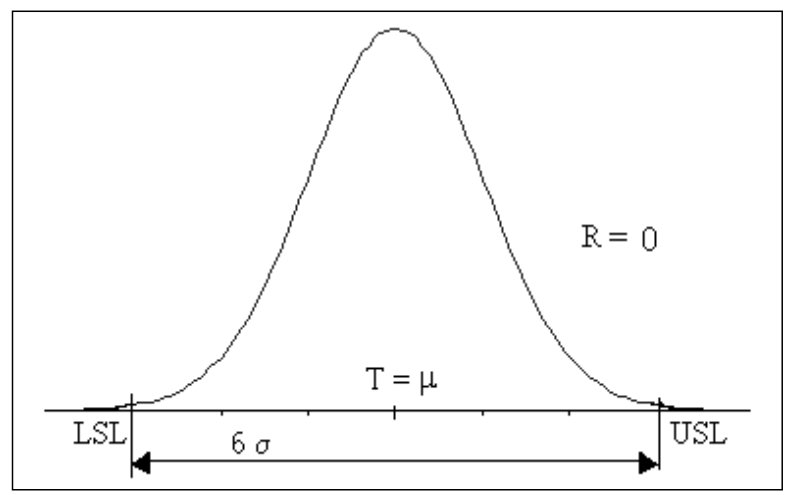

Figure 5 - Robustness $R=0$

Aside from robustness, there are other reasons why the capability index should be well above 1 . To give an example, let us assume that an aggregate is made up of 400 components, each of which is produced with the philosophy that it is enough to lie within the tolerance limits, i.e. $C_{p k}=1$. Then $N C$, or the probability that a part is outside the tolerance, is 0.0027 , but the probability of at least one part being outside the tolerance is, under the binomial model:

$$
P=1-\left(\begin{array}{c}
400 \\
0
\end{array}\right) \cdot 0.0027^{0} \cdot(1-0.0027)^{400}=0.6609
$$

There is a 66.09 per cent chance that at least one component will be an $N C$, and the aggregate will not function properly! By comparison, for $C_{p k}=1.33$, the $N C$ is 0.000066 and the probability of having at least one of the 400 components malfunctioning is:

$$
P=1-\left(\begin{array}{c}
400 \\
0
\end{array}\right) \cdot 0.000066^{0} \cdot(1-0.000066)^{400}=0.026
$$

The probability of having a defective aggregate is only 0.026 in this case!

\section{EVALUATION OF A CAPABILITY INDEX}

Denoting by $\hat{C}_{p m}$ the estimate of the population index $C_{p m}$, the two indices are naturally not the same, generally speaking. The customer demands the $C_{p m}$, the supplier can offer only the estimate unless it checks the whole production, which is something that usually exists only in theory. When testing significance of the estimate, given a probability $p$ and a number of measurements $n$, we ask the question what its value must be so that the $C_{p m}$ attains a required level $C$. Thus, 
we are looking for a certain value $\hat{C}_{p m}$ (min). For the most frequently used indices $C_{p}, C_{p k}, C_{p m}, C_{p m}^{*}$ and $C_{p m k}$, this problem is tackled in various ways. For instance, Tošenovský (2006) with the help of Capa follows the procedure described in Tab. 2. The table shows different ways of evaluation of the five indices. In practice, such an evaluation is unfortunately seldom performed.

Table 2 - Evaluation of Significance for Selected Capability Indices

\begin{tabular}{|l|l|}
\hline Index & Evaluation \\
\hline$C_{p}$ & Correction (Lewis, 1991) \\
\hline$C_{p k}$ & Correction (Lewis, 1991) \\
\hline$C_{p m}$ & Test $+\hat{C}_{p m}(\min )($ Chan, Cheng and Spiring, 1988) \\
\hline$C_{p m}^{*}$ & Test $+C_{p m}^{*}(\min )($ Chan, Cheng and Spiring, 1988) \\
\hline$C_{p m k}$ & Test (Pearn and Lin, 2002) \\
\hline
\end{tabular}

If an index is not calculated from the population, it is overestimated. The smaller the data sample, the more severe the overestimation, as shown in Tab. 3 and Fig. 6. To correct such an index means to remove the overestimation. The corresponding procedure is described in Lewis (1991).

Table 3 - Overestimation of the Indices $C_{p}$ and $C_{p k}$

\begin{tabular}{|c|c|c|}
\hline \multirow{2}{*}{ Sample Size } & \multicolumn{2}{|c|}{ Overestimation (\%) } \\
\cline { 2 - 3 } & $C_{p}$ & $C_{p k}$ \\
\hline 40 & 19 & 24 \\
\hline 50 & 17 & 21 \\
\hline 60 & 15 & 19 \\
\hline 70 & 14 & 18 \\
\hline 80 & 13 & 17 \\
\hline 90 & 12 & 16 \\
\hline 100 & 12 & 15 \\
\hline 150 & 10 & 12 \\
\hline
\end{tabular}

For instance, for $n=150$, the amount of overestimation is $10 \%$ for $C_{p}$ and $12 \%$ for $C_{p k}$. 


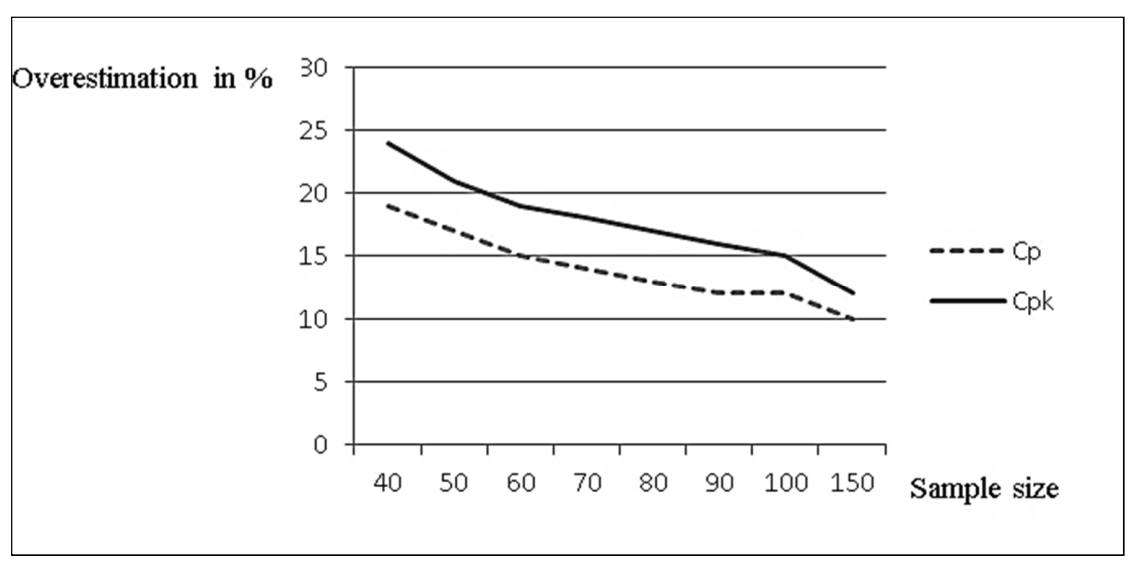

Figure 6-Overestimation in per Cent of $C_{p}$ and $C_{p k}$ with Respect to $n$

To make a more exact judgement about the significance of the sample indices, one should perform a statistical test. Nevertheless, such a test only gives information on whether the estimated index is or is not sufficiently high, and so, as mentioned earlier, it is also useful to calculate $\hat{C}_{p m}(\min )$ or similar characteristics.

Tab. 4 shows how $\hat{C}_{p m}(\min )$ depends on the sample size $n$, significance level $p$ and the required value of $C_{p m}$.

Table 4 - Values of $\hat{C}_{p m}(\min )$

\begin{tabular}{|c|c|c|c|c|c|c|}
\hline & \multicolumn{6}{|c|}{ Required values of the population index } \\
\hline & \multicolumn{2}{|c|}{$C p m=1.0$} & \multicolumn{2}{c|}{$C p m=1.33$} & \multicolumn{2}{c|}{$C p m=1.67$} \\
\hline $\mathrm{n}$ & $p=0.95$ & $p=0.99$ & $p=0.95$ & $p=0.99$ & $p=0.95$ & $p=0.99$ \\
\hline 10 & 1.56 & 1.97 & 2.11 & 2.62 & 2.66 & 3.28 \\
\hline 50 & 1.19 & 1.29 & 1.59 & 1.72 & 2.00 & 2.15 \\
\hline 75 & 1.15 & 1.23 & 1.53 & 1.63 & 1.93 & 2.04 \\
\hline 100 & 1.13 & 1.19 & 1.50 & 1.58 & 1.89 & 1.98 \\
\hline
\end{tabular}

For instance, given the sample size of 10, the required value of 1.33 and the degree of reliability 0.95 , the estimated index must attain or exceed the value 2.11, whereas for $n=100$, the value 1.50 will suffice. If the tables on $\hat{C}_{p m}$ (min) are available, they will only list specific options for $C_{p m}$, as shown in Tab. 4. The tables are also given for $n$ ranging from 3 to 100 and for $p=0.9$ or $p=0.95$ or $p$ $=0.99$. To make similar calculations of $\hat{C}_{p m}(\min )$ for any $n, p$ and $C_{p m}$, a special software must be used (Tošenovský, 2006). 


\section{MULTIVARIATE INDICES}

The multivariate indices are used if:

a) more operations are performed on a product, so that when evaluating the process (or the sequence of operations), more quality characteristics are observed, or

b) a product is made up of several components, each of which possesses a quality characteristic, and the product is evaluated as a whole.

When more quality characteristics are observed, it is not recommended to evaluate them separately with the aforementioned indices. When they are evaluated separately, then:

a) the process is not assessed as a whole, individual operations or components are evaluated instead,

b) if the characteristics are dependent, then not even a single operation is assessed, as the effect of the other operations is not factored into such a calculation.

In these cases, special multivariate capability indices were designed as well as graphical methods. The multivariate indices are denoted $M C_{p}, M C_{p k}, M C_{p m}$. For an index to be selected for use, it must satisfy proper conditions (see Tab. 5).

Table 5 - Conditions to Be Met by Multivariate Indices

\begin{tabular}{|l|l|}
\hline \multicolumn{1}{|c|}{$M C_{p}, M C_{p m}$} & \multicolumn{1}{|c|}{$M C_{p k}$} \\
\hline $\begin{array}{l}\text { Normally distributed quality characteristics with } \\
\text { two-sided tolerances. They do not have to be } \\
\text { independent. }\end{array}$ & $\begin{array}{l}\text { Independent quality characteristics } X_{i} \text { (this also } \\
\text { concerns attributes) with any type of tolerance. } \\
\text { Normality is not necessary. }\end{array}$ \\
\hline
\end{tabular}

To get the idea about the indices, we illustrate the situation with the bivariate version of the $M C_{p}$ index. Let there be two observed quality characteristics $X_{1}$, $X_{2}$, and let the corresponding random vector $\left(X_{1}, X_{2}\right)$ has the normal distribution $N(\mu, V)$. Further, let $T_{i}$ be the target value for $X_{i}, L S L_{i}$ be its lower specification limit and $U S L_{i}$ be its upper specification limit. The region of admissibility is then represented by a tolerance rectangle defined by the values $L S L_{1}$ and $U S L_{1}$ on the $X_{1}-$ axis and the values $L S L_{2}$ and $U S L_{2}$ on the $X_{2}-$ axis in the plane (Fig. 7, Fig. 8). 


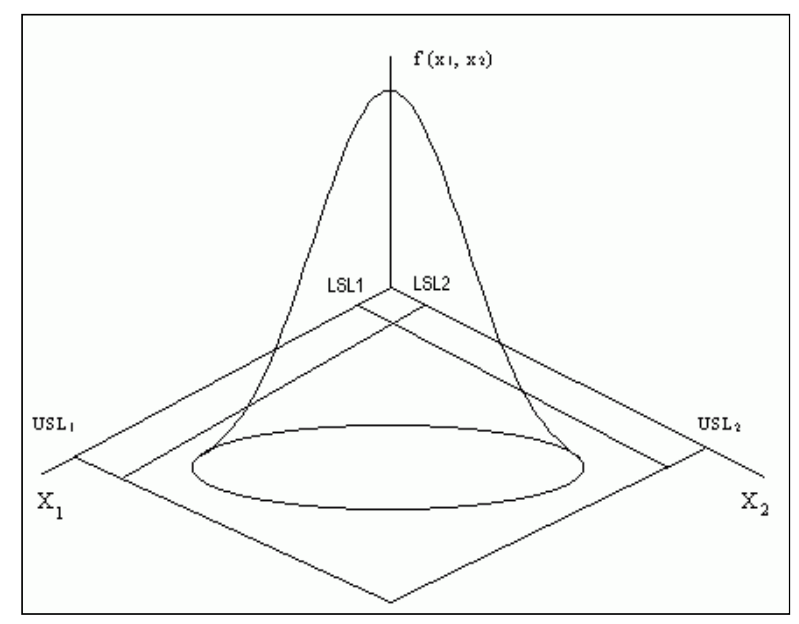

Figure 7 - A Bivariate Normal Distribution for $\left(X_{1}, X_{2}\right)$

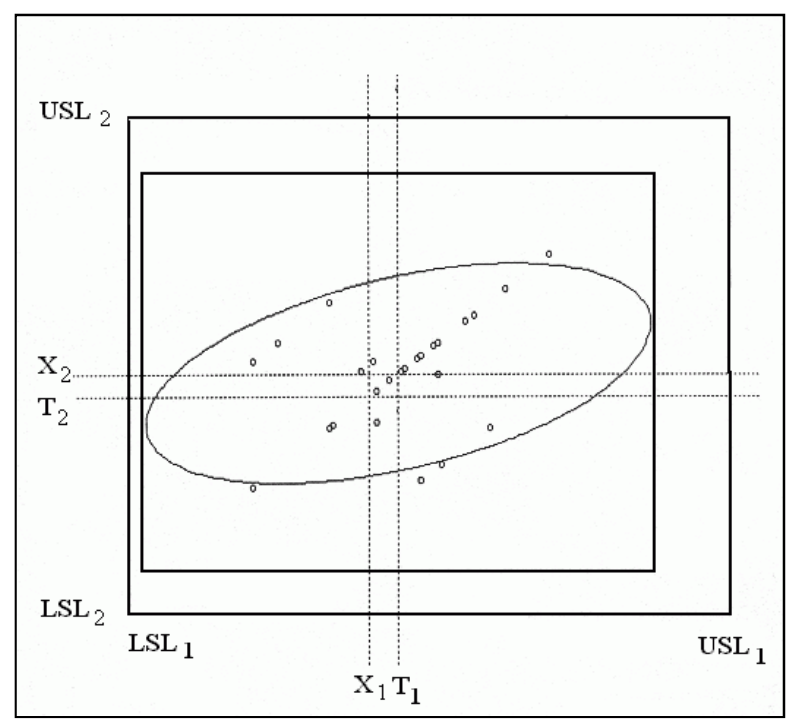

Figure $8-A$ View of the Plane $X_{1} \times X_{2}$

The target values $T_{1}$ and $T_{2}$ are the coordinates of the center of specification $T$, the actually achieved average values $\bar{X}_{1}, \bar{X}_{2}$ are the coordinates of the point $\bar{X}$. The distance between $T$ and $\bar{X}$ suggests the amount of process decetralization. Fig. 8 shows measurements and the ellipse attained by making a cut through the graph of the bivariate normal density $f\left(x_{1}, x_{2}\right)$ (Fig. 7), the cut being parallel to the plane $X_{1} \times X_{2}$. 
The sets of points at which the density is constant satisfy the equation:

$$
(\bar{X}-T)^{T} V^{-1}(\bar{X}-T)=\chi_{2}^{2}(\alpha)
$$

where $\bar{X}=\left(\begin{array}{l}\bar{X}_{1} \\ \bar{X}_{2}\end{array}\right), T=\left(\begin{array}{l}T_{1} \\ T_{2}\end{array}\right)$ and $V=\left(\begin{array}{ll}\sigma_{1}^{2} & \sigma_{12} \\ \sigma_{12} & \sigma_{2}^{2}\end{array}\right)$ is the variance matrix of $\left(X_{1}, X_{2}\right)$.

When working with three quality characteristics $X_{1}, X_{2}, X_{3}$, the formula represents a rotational ellipsoid.

Fig. 10 and Fig. 11 are analogies. The figures represent the projection of the tangent parallelepiped of the ellipsoid onto a selected plane $X_{i} \times X_{j}$. The plane is depicted as a rectangle together with the specification rectangle for the variables $X_{i}$ and $X_{j}$. The depicted ellipse is not a projection of the ellipsoid. It is a projection of its cut through the plane which is parallel to the plane $X_{i} \times X_{j}$ and runs through the center of the ellipsoid $\bar{X}=\left(\bar{X}_{1}, \bar{X}_{2}, \bar{X}_{3}\right)$. Since the cut is led through the center, the tangent parallelepiped may not touch the ellipse. In the case of three quality characteristics, the projection can be made onto the planes $X_{1} \times X_{2}, X_{1} \times X_{3}$ and $X_{2} \times X_{3}$.

The bivariate index $M C_{p}$ is defined similarly as $C_{p}$, i.e. as the ratio of the area that should contain measurements of the observed quality characteristics (the tolerance area) and the area that actually contains the measurements. While the interval of the length $6 \sigma$ stands for the true location of measurements in the $C_{p}$ case, the bivariate index $M C_{p}$ utilizes the ellipse or rectangle skirting the edges of this ellipse in the plane $X_{1} \times X_{2}$ (the tangent rectangle). In the formula (8) for the bivariate index, it is defined by the points $L_{1}, U_{1}, L_{2}, U_{2}$ :

$$
M C_{p}=\frac{\left(U S L_{1}-L S L_{1}\right)\left(U S L_{2}-L S L_{2}\right)}{\left(U_{1}-L_{1}\right)\left(U_{2}-L_{2}\right)}
$$

Similarly, for the k-dimensional index $M C_{p}, k>2$, we observe and evaluate:

a) the process centralization, using the so-called Hotelling's statistic (Hubele, Shahriari and Cheng, 1991),

b) the multivariate capability index (Pearn and Lin, 2002):

$$
M C_{p}=\frac{\prod_{i=1}^{k}\left(U S L_{i}-L S L_{i}\right)}{\prod_{i=1}^{k}\left(U_{i}-L_{i}\right)}
$$


While, for instance, Kotz and Johnson (1993) use in the denominator of $M C_{p}$ the volume of the rotational ellipsoid, (9) calculates the volume of the tangent parallelepiped, defined by the numbers $U_{i}, L_{i}$. The required limits $U_{i}, L_{i}$ can be read from Fig. 10 and Fig. 11 (software Capa used in this example):

c) the characteristic $M$ that identifies outliers (Hubele, Shahriari and Cheng,

$$
\text { 1991) is } M=\max \left\{1, \frac{\left|U_{1}-L S L_{1}\right|}{U S L_{1}-L S L_{1}}, \frac{\left|L_{1}-U S L_{1}\right|}{U S L_{1}-L S L_{1}}, \ldots, \frac{\left|U_{k}-L S L_{k}\right|}{U S L_{k}-L S L_{k}}, \frac{\left|L_{k}-U S L_{k}\right|}{U S L_{k}-L S L_{k}}\right\}
$$

$M$ should be less than one. For two variables $X_{1}, X_{2}$, we have:

$$
M=\max \left\{1, \frac{|U 1-L S L 1|}{U S L 1-L S L 1}, \frac{|U S L 1-L 1|}{U S L 1-L S L 1}, \frac{|U 2-L S L 2|}{U S L 2-L S L 2}, \frac{|U S L 2-L 2|}{U S L 2-L S L 2}\right\}
$$

Fig. 9 shows various limits used to calculate the characteristic.

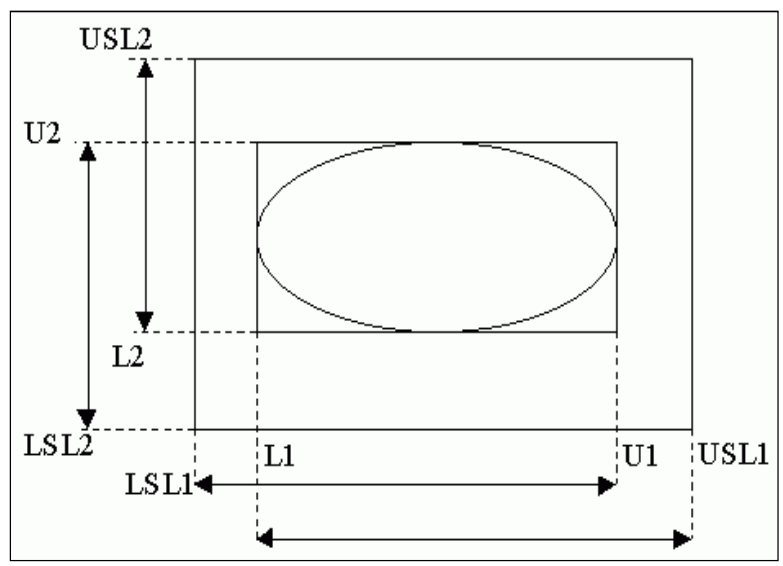

Figure 9-Parameters for the $M$ Characteristic

To give an example, if we are to calculate the $M C_{p}$ index for a process with three quality characteristics $X_{1}, X_{2}, X_{3}$, obtaining the data by examining the graphs, the procedure will be as follows:

Process Specifications:

$$
\begin{aligned}
& U S L_{1}=240, L S L_{1}=100, U S L_{1}-L S L_{1}=240-100=140 ; \\
& U S L_{2}=80, L S L_{2}=20, U S L_{2}-L S L_{2}=80-20=60 ; \\
& U S L_{3}=24, L S L_{3}=11, U S L_{3}-L S L_{3}=24-11=13 .
\end{aligned}
$$


The limits $U_{i}, L_{i}$, necessary for the calculation of $M C_{p}$, can be found in Fig. 10 and Fig. 11. For $X_{3}$ and $X_{1}$, we have from Fig. 10:

$L_{3}=11.311, U_{3}=24.128$

$L_{1}=114.03, U_{1}=240.36$;

$U_{3}-L_{3}=24.128-11.311=12.817$.

For $X_{1}$ and $X_{2}$, we have from Fig. 11:

$L_{1}=114.03, U_{1}=240.36$;

$L_{2}=32.447, U_{2}=72.217$;

$U_{1}-L_{1}=240.36-114.03=126.33$;

$U_{2}-L_{2}=72.217-32.447=39.77$.

The numerator of $M C_{p}$ contains the multiplication of the differences $U S L_{i}-L S L_{i}$, the denominator involves the multiplication of the differences $U_{i}-L_{i}$, therefore:

$$
M C_{p}=\frac{140 \cdot 60 \cdot 13}{126.33 \cdot 39.77 \cdot 12.82}=1.695
$$

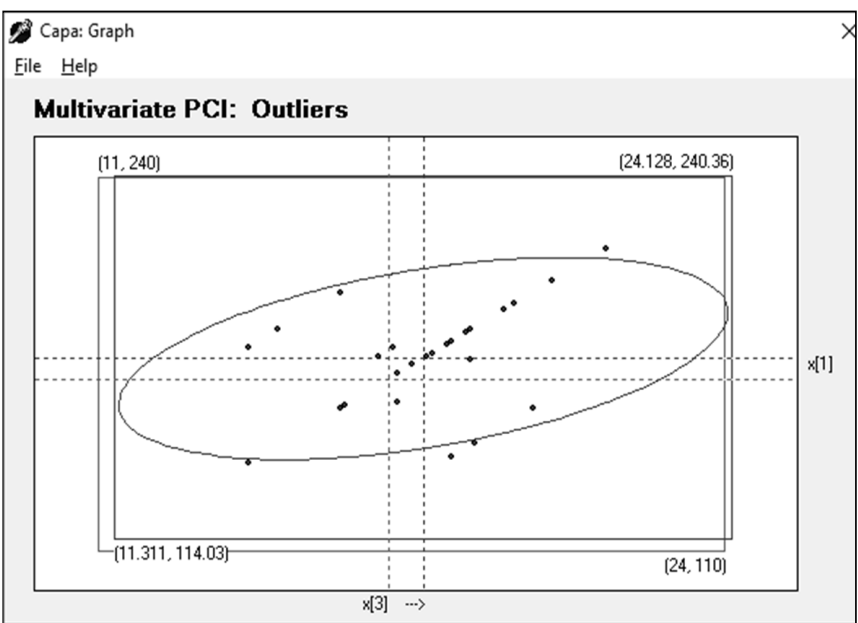

Figure 10-A Cut for given Values of $X_{1}$ and $X_{3}$ 


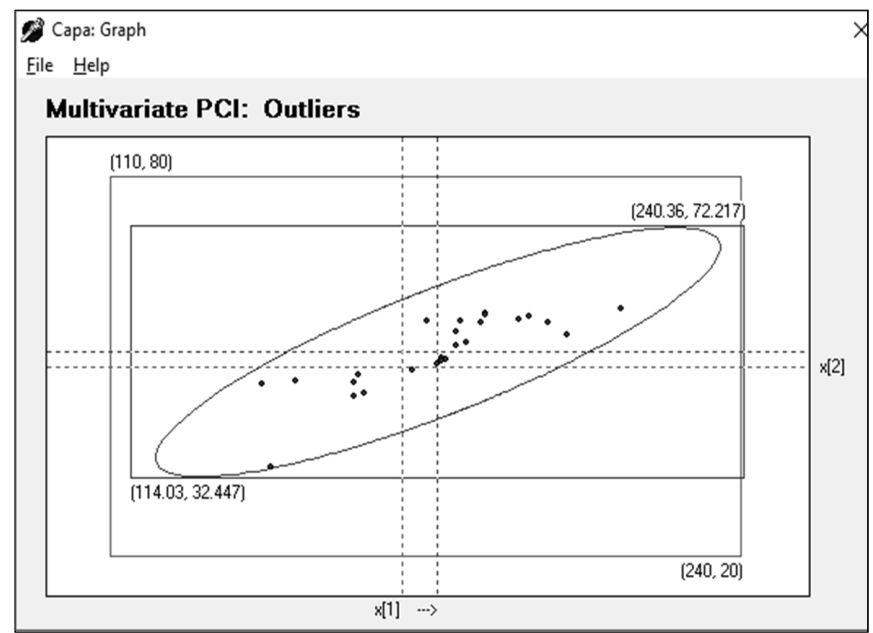

Figure 11-A Cut for given Values of $X_{1}$ and $X_{2}$

The graphical representation shown in Fig. 10 and Fig. 11 enables us to get the idea about:

a) the extent of centralization,

b) the extent to which the specification rectangle is exploited,

c) the location of the ellipse inside the rectangle,

d) the specification and tangent rectangles, needed for $M C_{p}$,

e) whether any measurements happen to be outside the specification rectangle.

Working with a multivariate index, Fig. 11 can be used to assess three attributes: $M C_{p}$, centralization and outliers (Hubele, Shahriari and Cheng, 1991). Fig. 12 represents different views of the plane $X_{1} \times X_{2}$ : the minus sign means the observed criterion is not compliant, the plus signs means it is compliant (the criteria are: $M C_{p}, X=T$, inequality $M<1$ ). 


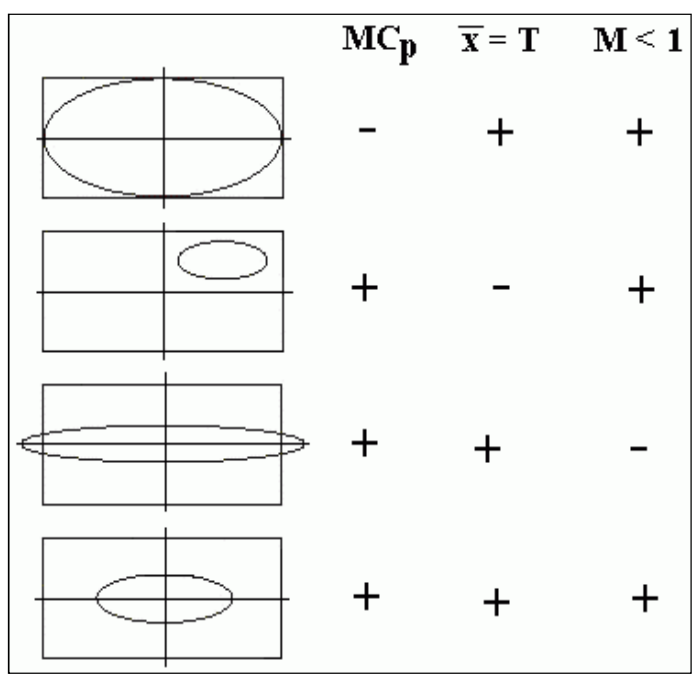

Figure 12 - Different Scenarios for Three Observed Criteria

\section{A ROBUST VERSION OF THE CPK INDEX}

Although data are analysed for possible outliers prior to capability index calculation, the data can be subsequently wrongly inserted in a computer, which can significantly alter the capability index being calculated. When calculating a univariate or multivariate capability index, the sample average and variance are also calculated. If the data is contaminated with outliers, moment characteristics will be biased, and so will the capability indices. This can be prevented with the following (robust) adaptation of $C_{p k}$ : the median $\mathrm{M}$ is used instead of the average and the median of absolute differences MAD instead of variance, in other words $\mathrm{MAD}=\operatorname{med}\left(\left|\mathrm{x}_{\mathrm{i}}-\operatorname{med} \mathrm{x}_{\mathrm{i}}\right|\right)$.

$R C_{p k}$ will then be calculated according to the formula:

$$
R C p k=\min \left(\frac{U S L-M}{3 \cdot M A D}, \frac{M-L S L}{3 \cdot M A D}\right)
$$

We shall now illustrate the use of this robust version. For the data 12, 15, 14, 11, 10 and the specification $\mathrm{USL}=18.61, \mathrm{LSL}=6.19, \mathrm{~T}=12.4$, let us calculate $C_{p k}$ and $R C_{p k}$ as follows:

a) $C_{p k}$ in the standard way;

b) $C_{p k}$ with the false value $\mathrm{x}_{5}=1.0$ instead of 10 ;

c) $R C_{p k}$ for flawless data;

d) $R C_{p k}$ for flawed data: $\mathrm{x}_{5}=1.0$ instead of 10. 
The results are:

a) $C_{p k}=0.998$ (calculated with Capa);

b) $C_{p k}=0.26$ (average is 10.6; standard deviation equals 5.59);

c) $\mathrm{M}=12, \mathrm{MAD}=2$,

$$
\begin{aligned}
& R C p U=\frac{U S L-M}{3 \cdot M A D}=\frac{18.61-12}{3.2}=1.102 ; \quad R C p L=\frac{M-L S L}{3 \cdot M A D}=\frac{12-6.19}{3.2}=0.968 \\
& R C p k=0.968
\end{aligned}
$$

d) $\mathrm{M}=12$ and $\mathrm{MAD}=2$, i.e. the same values as for the correct data, and so the index remains the same.

For the correct data, $C_{p k}$ and $R C_{p k}$ do not differ significantly. For the wrong data, $R C_{p k}$ keeps its value attained for the correct data.

Knowledge of multivariate data is also used in other calculations, such as regression, where robust techniques are exploited, as well Kutner, Nachtsheim and Neter (2014). A simple and efficient procedure is, for instance, that of obtaining regression coefficients with the generalized-least-squares formula $\mathbf{b}=$ $\left(\mathrm{X}^{\mathrm{T}} \mathrm{WX}\right)^{-1} \mathrm{X}^{\mathrm{T}} \mathrm{WY}$, where $\mathrm{W}$ is a matrix of weights. It is a diagonal matrix with elements w. Two widely used weight functions are the Huber and bisquare weight functions. According to Huber, $\mathrm{w}=1.345 / \mathrm{u}, \mathrm{u}=\mathrm{e} / 4.6683$, where e is a residual from the classical least-squares estimation method. These techniques, studied in students' SGS projects, for instance, proved to be robust.

\section{CONCLUSION}

The aim of the paper was to show how educational process in the field of process evaluation can be complemented with graphical and numerical illustrations of selected chapters on this subject. We believe that visualization and numerical examples are a way to make statistical methods more popular. It is also very convenient to provide students with enough material for their self-training, with a software that provides quick solutions, is up to date in the field and can be used for real-life problems, if possible. We have prepared a 380-pages long training manual with the most frequently occurring real-life problems, illustrated with graphs and solutions. The solutions can also be obtained with the software Capa that is part of the manual (Tošenovský, 2006). Our experience is such that knowledge of modern methods of capability evaluation is needed not only for producers, but also for customers. In the latter case, lack of knowledge often leads to situations when customers require inadequate means of capability assessment. 


\section{ACKNOWLEDGEMENTS}

This paper was prepared under the specific research projects No. SP2018/109, SP2019/62 and SP2019/129 conducted at the Faculty of Materials Science and Technology, VŠB-TU Ostrava, with a support from the Ministry of Education of the Czech Republic.

\section{REFERENCES}

Bothe, D.R., 2000. Composite capability index for multiple product Characteristic. Quality Engineering, [e-journal] 12(2), pp.253-258. https://doi.org/10.1080/08982119908962582.

Clements, J.A., 1989. Process capability calculations for non-normal Distributions. Quality Progress, 22, pp.95-100.

Holmes, D.S and Mergen, A.E., 1995. An alternative method to test for randomness of a process. Quality and Reliability Engineering International, 11(3), pp.171-174.

Hubele, N.F., Shahriari, H. and Cheng, C.S., 1991. A bivariate process capability vector in statistics and design in process control. Statistical Process Control in Manufacturing, pp.299-310.

Chan, L.K., Cheng, S.W. and Spiring, F.A., 1988. A new measure of process capability: Cpm. Journal of Quality Technology, [e-journal] 20(3), pp.162-175. https://doi.org/10.1080/00224065.1988.11979102.

Kotz, S. and Johnson, N., 1993. Process capability indices. London: Chapman \& Hall.

Krishnamoorthi, K.S., 1990. Capability indices for process subject to unilateral and positional tolerance. Quality Engineering, [e-journal] 2(4), pp.461-471. https://doi.org/10.1080/08982119008962740.

Kutner, M.H., Nachtsheim, CH.J. and Neter, J., 2014. Applied Linear Regresion Models. New York: McGraw-Hill.

Lewis, S.S., 1991. Process capability estimates from small samples. Quality Engineering, 3(3), pp.381-394. https://doi.org/10.1080/08982119108918865.

Pearn, W.L. and Kotz, S., 1995. Application of Clements' method for calculating second and third generation process capability indices for non-normal Pearson populations. Quality Engineering, [e-journal] 7(1), pp.139-145. https://doi.org/10.1080/08982119408918772.

Pearn, W.L. and Lin, P.C., 2002. Computer program for calculating the p-value in testing process capability index Cpmk. Quality and Reliability Engineering, [e-journal] 18(4), pp.333-342. https://doi.org/10.1002/qre.465. 
Phillips, G.P., 1995. Target ratio simplifies capability index system, makes it easy to use Cpm. Quality Engineering, [e-journal] 7(2), pp.299-313. https://doi.org/10.1080/08982119408918785.

Schneider, H., Pruett, J. and Lagrange, C., 1996. Uses of process capability indices in the supplier certification process. Quality Engineering, [e-journal] $8(2)$, pp.225-235.

Tošenovský, J., 2006. Capa. [computer program] Josef Tošenovský. Available at: <josef.tosenovsky@vsb.cz> [01 March 2006].

Zgodavova, K. and Slimak, I., 2008. Advanced Improvement of Quality. In: DAAM, 19th International Symposium of the Danube-Adria-Association-forAutomation-and-Manufacturing Location. Trnava, Slovakia, pp.1551-1552.

\section{ABOUT AUTHORS}

Josef Tošenovský - The author is a full professor at VŠB-Technical university of Ostrava, specializing in probability, statistics and econometrics. In 1988 he became an associate professor in mathematics and in 2001 a professor in Management of Industrial Systems. E-mail: josef.tosenovsky@vsb.cz, Author's ORCID: https://orcid.org/0000-0003-4684-1369.

Filip Tošenovský - The author is an assistant professor at VŠB-Technical university of Ostrava, specializing in probability, statistics, econometrics and operations research. E-mail: filip.tosenovsky@vsb.cz, Author's ORCID: https://orcid.org/0000-0003-3946-7815.

(C) 2019 by the authors. Submitted for possible open access publication under the terms and conditions of the Creative Commons Attribution (CC-BY) license (http://creativecommons.org/licenses/by/4.0/). 